\title{
Influence hydrothermale dans les sédiments de fosses de la mer Rouge par l'étude de quelques éléments traces
}

\author{
Marie-Claire PIERRET $^{\text {a*}}$, Norbert CLAUER ${ }^{a}$, Gérard BLANC ${ }^{b}$ \\ ${ }^{\text {a }}$ Centre de géochimie de la surface, école et observatoire des sciences de la terre, UMR-CNRS 7517, 1, \\ rue Blessig, F-67084 Strasbourg cedex, France \\ ${ }^{\mathrm{b}}$ Département de géologie et d'océanographie, UMR-CNRS 5805, avenue des Facultés, \\ F-33405 Talence cedex, France
}

Reçu le 24 janvier 2000, reçu en forme révisée le $11^{\text {er }}$ juillet 2000 , accepté le 12 juillet 2000

\begin{abstract}
Hydrothermal impact on sediments of Red-Sea deeps by the study of some trace metals. The $\mathrm{Cu}, \mathrm{Ni}$, $\mathrm{Fe}, \mathrm{Cr}$ and $\mathrm{Zr}$ concentrations were evaluated to study the influence of hydrothermal activity on the sediments of six Red-Sea deeps. Comparison between the $\mathrm{Ni} / \mathrm{Fe}$ and $\mathrm{Cu} / \mathrm{Fe}$ ratios supports distinction between hydrothermal and non-hydrothermal sediments, whereas anti-correlation between $\mathrm{Zr}$ and $\mathrm{Cr}$ concentrations results from hydrothermal influence. The results allow us to envisage that the sediments of the Suakin Deep did not undergo a hydrothermal activity and that this hydrothermal influence was weak in the Valdivia Deep. The sediments of Port-Soudan Deep recorded a hydrothermal activity, whereas this activity was the strongest in the Thetis and Atlantis II deeps. C) 2000 Ifremer/CNRS/IRD/Éditions scientifiques et médicales Elsevier SAS
\end{abstract}

\section{sediment / Red sea / deeps / hydrothermalism / metals}

Résumé - Les teneurs en $\mathrm{Cu}, \mathrm{Ni}, \mathrm{Fe}, \mathrm{Cr}$ et $\mathrm{Zr}$ servent ici à évaluer l'influence d'une activité hydrothermale sur les sédiments de six fosses de la mer Rouge. Ainsi, l'enrichissement en fer (Fe) et en cuivre $(\mathrm{Cu})$ des fluides hydrothermaux provenant du lessivage de la croûte océanique se retrouve dans les sédiments hydrothermaux alors que le nickel (Ni) est essentiellement apporté par l'eau de mer. La comparaison des rapports $\mathrm{Ni} / \mathrm{Fe}$ et $\mathrm{Cu} / \mathrm{Fe}$ représente donc un bon paramètre de discrimination entre les sédiments biodétritiques et ceux d'origine hydrothermale. Le chrome $(\mathrm{Cr})$ et le zirconium $(\mathrm{Zr})$ proviennent essentiellement du matériel détritique dans les sédiments marins normaux et sont corrélés, alors que l'absence de corrélation entre ces deux métaux est un indice d'une activité hydrothermale. Les résultats cumulés montrent que les sédiments de la fosse Suakin n'ont pas subi d'influence hydrothermale, et que celle-ci est très faible dans la fosse Valdivia. Les sédiments de la fosse Port-Soudan ont aussi enregistré les effets d'arrivées hydrothermales, mais l'activité hydrothermale est la plus importante et la plus nette dans la fosse Thétis et, surtout, dans celle d'Atlantis II. (C) 2000 Ifremer/CNRS/IRD/ Éditions scientifiques et médicales Elsevier SAS

sédiment / mer Rouge / fosses / hydrothermalisme / métaux

\section{INTRODUCTION}

La mer Rouge est un océan étroit qui occupe le rift résultant de l'éloignement des plaques arabique et

* Correspondance et tirés à part : africaine. Elle offre la possibilité d'étudier la transiE-mail address: pierret@illite.u-strasbg.fr (M.-C. Pierret). tion du rift continental vers un rift océanique. La 
croûte océanique est continue seulement dans la partie méridionale de la vallée axiale (figure 1); au delà, elle devient discontinue et la partie centrale comprend une vingtaine de fosses, assimilées à des cellules d'océanisation (Bäcker et Schoell, 1972). Ces dépressions bathymétriques représentent une des grandes originalités de la mer Rouge et se caractérisent par la présence de saumures chaudes et/ou de sédiments métallifères. Les processus responsables de la formation de ces saumures et sédiments ont été essentiellement étudiés dans Atlantis II, la fosse la plus importante et la plus minéralisée, et sont associés à l'activité hydrothermale qui y règne (Anschutz et Blanc, 1993a,b, 1995a,b; Bäcker et Richter, 1973; Blanc et al., 1998; Degens et Ross, 1969 ; Pottorf et Barnes, 1983 ; Zierenberg et Schanks, 1986). L'exis-

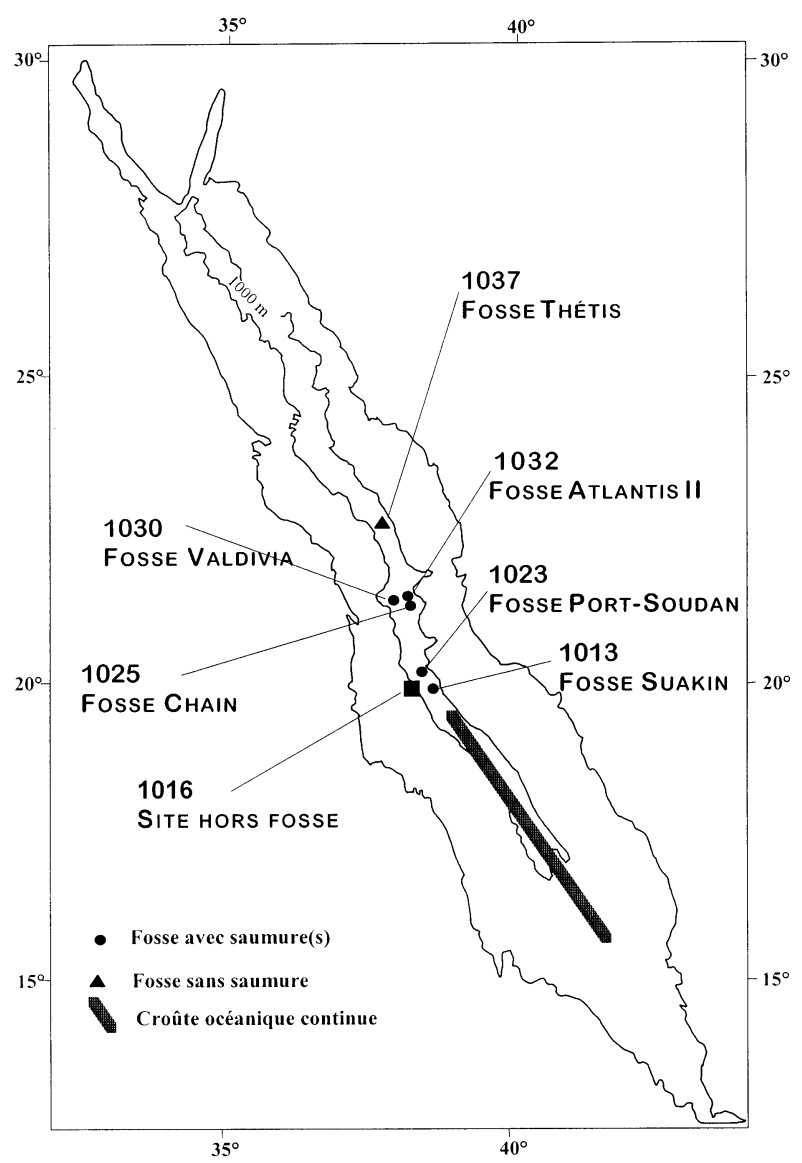

Figure 1. Carte de la mer Rouge avec localisation des différents sites échantillonnés et des carottes correspondantes.

Figure 1. Map of the Red Sea with location of the different sample sites and corresponding cores. tence des saumures est imputée au lessivage d'épais niveaux évaporitiques miocènes (principalement composés de halite et d'anhydrite) entraînant la formation de solutions salées plus denses que l'eau de mer et qui restent alors piégées dans ces fosses. Peu d'études ont été publiées sur des fosses autres que celles de la zone Atlantis II et peu de comparaisons ont été réalisées entre les différentes fosses.

En 1992, des sédiments et des saumures ont été échantillonnés pour la première fois dans plusieurs fosses de la partie médiane de la zone axiale de la mer Rouge au cours de la mission Redsed (fosses Suakin, Port-Soudan, Chain, Valdivia, Atlantis II et Thétis). Ce matériel permet d'étudier et de comparer plusieurs fosses, et ainsi de vérifier si l'influence hydrothermale est identique dans toutes les fosses de la mer Rouge et si les modèles de fonctionnement décrits pour la fosse Atlantis II sont applicables aux autres fosses. En fait, le taux de minéralisation varie d'une fosse à l'autre, ce qui implique de prendre en compte plusieurs processus de sédimentation dont les effets varient d'un site à l'autre (Pierret, 1998). Ainsi, à la sédimentation biodétritique habituelle de la mer Rouge se superpose une sédimentation chimique variable à partir des saumures. L'objectif de ce travail est d'étudier le comportement de certains éléments, comme les métaux $\mathrm{Fe}, \mathrm{Ni}, \mathrm{Cu}$ et $\mathrm{Cr}$ et le $\mathrm{Zr}$ dans les différents contextes de sédimentation qu'offrent ces six fosses échantillonnées de la mer Rouge, d'en déduire l'impact des manifestations hydrothermales et de les comparer.

\section{MATÉRIELS ET MÉTHODES}

Des saumures et des sédiments ont été collectés dans plusieurs fosses de la partie médiane de la mer Rouge (Thétis, Valdivia, Atlantis II, Chain, Port-Soudan et Suakin), dans le cadre de la mission Redsed effectuée en 1992 à bord du NO Marion Dufresne. Les sept carottes échantillonnées et utilisées dans ce travail, sont localisées sur la figure 1. Un carottage supplémentaire a été réalisé à environ dix kilomètres à l'ouest de Suakin, dans la plaine qui surplombe la fosse Suakin (carotte 1016). Les sédiments de cette carotte hors fosse reflètent le fond biodétritique de la mer Rouge et sont considérés comme la référence non hydrothermale. Dans la présente étude, vingt et un échantillons de sédiments de la carotte 1016 ont été 
analysés (14,4 $\mathrm{m}$ de longueur, site hors fosse), huit de la carotte 1025 (2,8 $\mathrm{m}$ de longueur, fosse Chain), seize de la carotte 1030 (11,6 m de longueur, fosse Valdivia), vingt de la carotte 1037 (6,2 m de longueur, fosse Thétis), vingt-deux de la carotte 1013 (5,8 m de longueur, fosse Suakin), dix-neuf de la carotte 1023 (6,3 m de longueur, fosse Port-Soudan) et cinquantetrois de la carotte 1032 (23,3 m de longueur, fosse Atlantis II).

Ces sédiments ont fait l'objet d'une étude minéralogique et géochimique détaillée (Pierret, 1998). Cet article présente seulement les teneurs en $\mathrm{Cu}, \mathrm{Ni}, \mathrm{Fe}$, $\mathrm{Cr}$ et $\mathrm{Zr}$ parce qu'ils représentent des discriminants potentiels de l'activité hydrothermale qui a pu exister dans le contexte des fosses étudiées.

Les échantillons ont été dessalés et séchés. Puis ces sédiments ont été broyés et leur composition minéralogique déterminée par diffraction des rayons X. Les concentrations en éléments majeurs, en éléments traces et en terres rares ont été acquises par fusion alcaline (Samuel et al., 1985). Le fer a été dosé par spectrométrie d'émission atomique à arc, $\mathrm{Cu}$ et $\mathrm{Ni}$ par ICP-AES, et les terres rares, $\mathrm{Zr}$ et $\mathrm{Cr}$ par ICPMS. La précision des analyses, évaluée grâce à des mesures de standards internationaux de sédiments marins (MAG1, SD01 et SD02), est inférieure ou égale à $5 \%$ pour tous les éléments. La reproductibilité des mesures est de l'ordre de 2 à $5 \%$. Les proportions relatives des minéraux ont été déterminées par compilation de deux méthodes: une basée sur l'intensité des pics rayons $\mathrm{X}$ et une qui conjugue les données minéralogiques et les concentrations chimiques. Ainsi, des profils lithostratigraphiques ont-ils été décrits pour chacune des fosses, permettant de différencier des unités lithologiques (Pierret, 1998 ; Pierret et al., 2000) (tableau I).

\section{RÉSULTATS}

Les teneurs en $\mathrm{Fe}, \mathrm{Ni}, \mathrm{Cu}, \mathrm{Cr}$ et $\mathrm{Zr}$ sont variables d'un site à l'autre, avec des concentrations en $\mathrm{Fe}$ comprises entre 2,5\% pour les sédiments biodétritiques normaux de la mer Rouge jusqu'à une moyenne de $50 \%$ dans l'unité U3 de la fosse Thétis. Les teneurs en $\mathrm{Ni}, \mathrm{Cu}, \mathrm{Cr}$ et $\mathrm{Zr}$ s'échelonnent entre respectivement $43,22,26$ et $13 \times 10^{-6}$ pour les valeurs les plus faibles et respectivement 273, 8165, 107 et
$158 \times 10^{-6}$ pour les plus élevées (tableau I). L'écart le plus important est observé pour le $\mathrm{Cu}$ avec un facteur de 378 entre les valeurs minimale et maximale, alors que celui-ci est compris entre 3 et 20 pour les autres éléments. La moyenne des vingt et un échantillons provenant $\mathrm{du}$ site hors fosse reflète le fond biodétritique normal de la mer Rouge sans apports hydrothermaux, avec des teneurs faibles en $\mathrm{Fe}, \mathrm{Ni}$ et $\mathrm{Cu}$ et un rapport $\mathrm{Ni} / \mathrm{Fe}$ élevé. Les sédiments hydrothermaux de la fosse Atlantis II se caractérisent par des teneurs en $\mathrm{Fe}$ et en $\mathrm{Cu}$ importantes associées à des teneurs en $\mathrm{Zr}$ faibles avec des rapports $\mathrm{Cu} / \mathrm{Fe}$ élevées (entre 35 et 510$)$ et $\mathrm{Ni} / \mathrm{Fe}$ faibles $(1,3$ à 8,8$)$. À l'opposée, les sédiments de la fosse Suakin ont les teneurs les plus faibles en $\mathrm{Cu}$ et les plus élevées en $\mathrm{Ni}$ et en $\mathrm{Zr}$ ce qui se traduit par des rapports $\mathrm{Cu} / \mathrm{Fe}$ faibles $(3,8$ à 5$)$ et $\mathrm{Ni} / \mathrm{Fe}$ élevés (17 à 33, tableau I). Les autres sédiments ont des comportements intermédiaires (figure 2). La figure $2 a$ illustre la différence entre le groupe des sédiments d'Atlantis II et les autres. Dans les fosses Thétis et Port-Soudan et dans l'unité U2 de Chain, des rapports $\mathrm{Ni} / \mathrm{Fe}$ bas sont associés à des rapports $\mathrm{Cu} / \mathrm{Fe}$ élevés, alors que les sédiments biodétritiques (carotte 1016) se caractérisent par un rapport $\mathrm{Ni} / \mathrm{Fe}$ plus important que le rapport $\mathrm{Cu} / \mathrm{Fe}$ (figure 2a). Dans le diagramme donnant la variation de $[\mathrm{Zr}]$ en fonction de $[\mathrm{Cr}]$, les sédiments des fosses Atlantis II et Thétis appartiennent à un domaine (espace grisé sur la figure 3) qui se différencie des autres points pour lesquels $\mathrm{Zr}$ et $\mathrm{Cr}$ sont mieux corrélés et où les teneurs en $\mathrm{Zr}$ sont plus importantes (figure 3).

\section{DISCUSSION}

\subsection{Signification des teneurs}

Lors de la circulation hydrothermale à travers la croûte océanique, les caractéristiques physicochimiques de l'eau de mer se modifient. Les interactions eau/roche diffèrent suivant la profondeur, et lorsque la zone de réaction est atteinte les métaux tels que $\mathrm{Fe}, \mathrm{Cu}, \mathrm{Zn}$ et $\mathrm{Pb}$ sont lessivés (Alt, 1995 ; Chiba, 1995) enrichissant le fluide hydrothermale en ces éléments (Von Damm, 1995). Ainsi, les sédiments associés à l'hydrothermalisme océanique, c'est-à-dire ceux qui se forment à proximité des zones de décharges des 


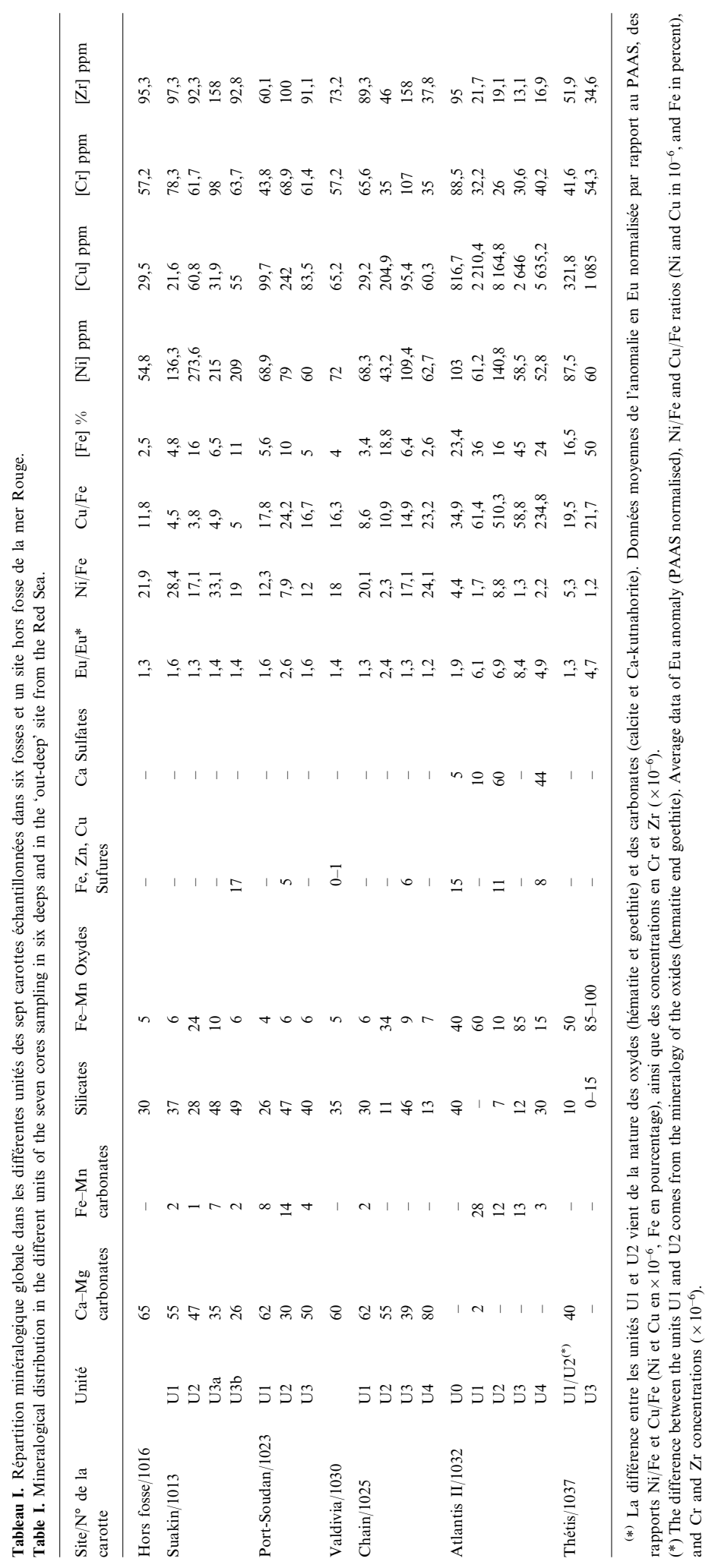



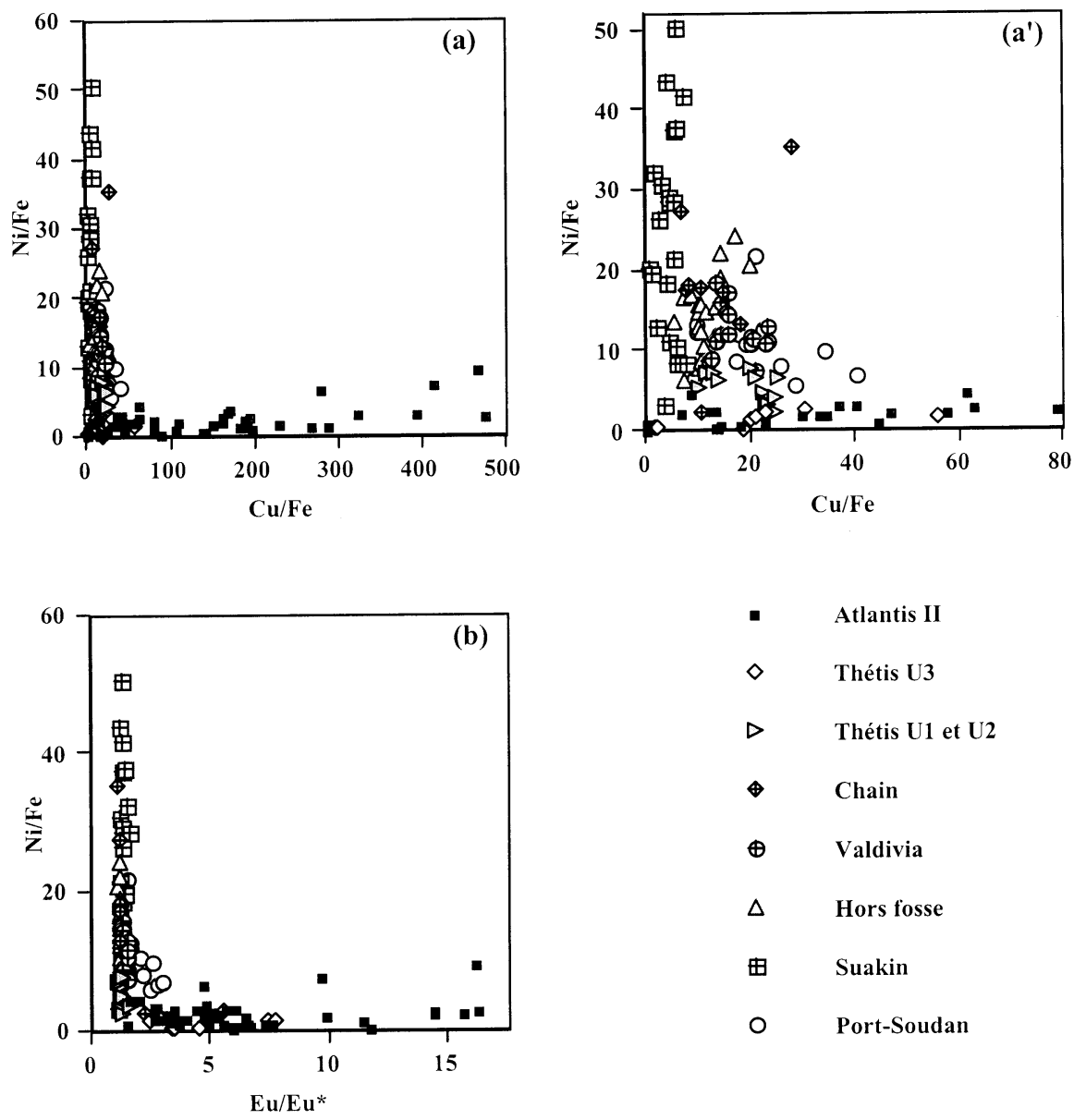

Figure 2. Étude des rapports $\mathrm{Cu} / \mathrm{Fe}$ et $\mathrm{Ni} / \mathrm{Fe}$. Les rapports sont donnés pour $\mathrm{Fe}$ en pourcentage et $\mathrm{Cu}$ et $\mathrm{Ni}$ en $\times 10^{-6}$ (a) Variations du rapport $\mathrm{Ni} / \mathrm{Fe}$ en fonction du rapport $\mathrm{Cu} / \mathrm{Fe}$, (a') même figure que la précédente mais avec dilatation de l'échelle et (b) variation du rapport $\mathrm{Ni} / \mathrm{Fe}$ en fonction de l'anomalie en Eu (normalisation par rapport au PAAS).

Figure 2. Study of the $\mathrm{Cu} / \mathrm{Fe}$ and $\mathrm{Ni} / \mathrm{Fe}$ ratios, $\mathrm{Fe}$ in percent and $\mathrm{Cu}$ and $\mathrm{Ni} \times 10^{-6}$. (a) $\mathrm{Ni} / \mathrm{Fe}$ ratio versus $\mathrm{Cu} / \mathrm{Fe}$ ratio, (a') same figure as precedent with an extending scale, and (b) $\mathrm{Ni} / \mathrm{Fe}$ ratios versus Eu anomaly (PAAS normalised).

fluides hydrothermaux, sont nettement enrichis en $\mathrm{Cu}, \mathrm{Fe}, \mathrm{Zn}$ et $\mathrm{Pb}$ par rapport aux sédiments marins normaux. Les teneurs en $\mathrm{Cu}$ y sont comprises entre 1 et $15 \%$, alors que les teneurs en $\mathrm{Ni}$ sont entre 2 et $50 \times 10^{-6}$ (Fouquet et al., 1991 ; Hannington et al., 1991, 1995 ; Haymond et Kastner, 1981 ; Hekinian et Fouquet, 1985; Marchig et al., 1982; Metz et al., 1988 ; Turner et al., 1993). En revanche, les teneurs en Ni sont environ trois fois plus importantes qu'en $\mathrm{Cu}$ dans l'eau de mer (Bruland et Franks, 1983); les organismes marins, ainsi que le matériel détritique comme les argiles, sont généralement plus riches en Ni qu'en Cu (Calvert et Pedersen, 1993; Collier et Edmond, 1983). Ainsi, Ni est plus concentré que $\mathrm{Cu}$ dans les sédiments marins et, en particulier, dans des conditions anoxiques. Ces éléments sont incorporés aux sédiments par adsorption, ou sous forme de sulfures dans des conditions anoxiques (Calvert et Pedersen, 1993). En contexte hydrothermal, Cu, Zn, $\mathrm{Pb}$ et $\mathrm{Fe}$ incorporés dans le sédiment précipitent depuis le fluide hydrothermal, alors que la teneur en $\mathrm{Ni}$ est principalement contrôlée par l'eau de mer (Hekinian et al., 1993; Metz et al., 1988), ce qui explique les faibles teneurs en $\mathrm{Ni}$ dans les dépôts hydrothermaux. Plus encore que les teneurs, les rapports de concentrations peuvent être discriminants. Les sédiments marins moyens ont des rapports $\mathrm{Ni} / \mathrm{Fe}$ et $\mathrm{Cu} / \mathrm{Fe}$ de respectivement 12,7 et 7,3 [calculés 
d'après les valeurs moyennes de (Metz et al., 1988)]. Les dépôts hydrothermaux (sulfures massifs, fumeurs noirs et blancs) et les sédiments hydrothermaux ont des rapports $\mathrm{Ni} / \mathrm{Fe}$ qui oscillent entre 0,2 et 2,5 et entre 0,4 et 1 , et des rapports $\mathrm{Cu} / \mathrm{Fe}$ entre 150 et 7000 , et entre 400 et 800 (Fouquet et al., 1991; Hannington et al., 1991, 1995 ; Haymond et Kastner, 1981 ; Hekinian et Fouquet, 1985; Marchig et al., 1982 ; Metz et al., 1988 ; Turner et al., 1993).

Comme il existe des sédiments métallifères à la fois diagénétiques et hydrothermaux, il a été proposé de différencier ces deux types à partir des valeurs en $\mathrm{Cr}$ et en $\mathrm{Zr}$ (Marchig et al., 1982). L'origine du $\mathrm{Cr}$ des sédiments non hydrothermaux se trouve dans le matériel continental; il est alors corrélé avec d'autres éléments traceurs du détritique tels que $\mathrm{Th}, \mathrm{Al}$ et $\mathrm{Zr}$. $\mathrm{Le} \mathrm{Cr}$ et le $\mathrm{Zr}$ sont, par contre, liés différemment dans les fluides hydrothermaux où $\mathrm{Cr}$ est plus enrichi que $\mathrm{Zr}$ (Turner et al., 1993). Les teneurs de ces deux éléments sont plus faibles dans les sédiments métallifères ayant une origine hydrothermale que dans des sédiments marins et ne sont pas corrélés (Blanc, 1994 ; Hekinian et al., 1993; Hekinian et Fouquet, 1985 ; Metz et al., 1988). En effet, les apports détritiques dans les sédiments se trouvent dilués en contexte hydrothermal par les minéraux précipitant du fluide hydrothermal et la corrélation $\mathrm{Cr} / \mathrm{Zr}$ liée au

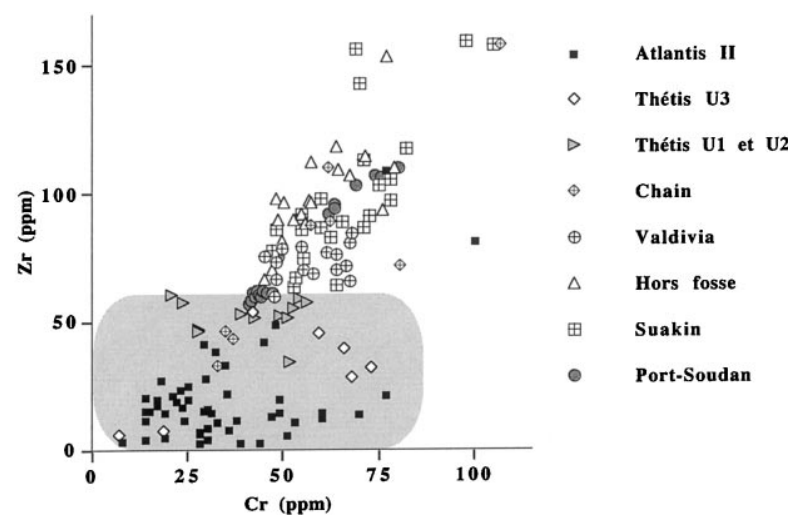

Figure 3. Variations de la teneur en $\mathrm{Zr}\left(\times 10^{-6}\right)$ en fonction de la teneur en $\mathrm{Cr}\left(\times 10^{-6}\right)$ dans les sédiments des fosses Atlantis II, Thétis, Chain, Valdivia, Suakin, Port-Soudan et dans le site hors fosse. La zone grisée représente le domaine des sédiments hydrothermaux.

Figure 3. $\mathrm{Zr}$ contents $\left(\times 10^{-6}\right)$ versus $\mathrm{Cr}$ contents $\left(\times 10^{-6}\right)$ in the sediments of Atlantis II, Thetis, Chain, Valdivia, Suakin and Port-Soudan deeps and in the 'out-deep' site. The grey field represent the area of hydrothermal sediments. détritique disparaît. Ainsi, une corrélation positive entre $\mathrm{Cr}$ et $\mathrm{Zr}$ reflète l'héritage sédimentaire détritique, et l'absence de relation entre $\mathrm{Cr}$ et $\mathrm{Zr}$, associé à des teneurs en $\mathrm{Cr}$ et surtout en $\mathrm{Zr}$ plus faibles que dans les sédiments marins moyens, est un indicateur d'une origine hydrothermale.

\subsection{L'origine des sédiments des fosses}

Les sédiments du site hors fosse représentent la signature biodétritique de la mer Rouge et se caractérisent par des rapports $\mathrm{Ni} / \mathrm{Fe}$ compris entre 6,2 et 24 , toujours supérieurs aux rapports $\mathrm{Cu} / \mathrm{Fe}$ compris entre 5,7 et 17,2 typiques de sédiments non hydrothermaux et par une corrélation entre $\mathrm{Cr}$ et $\mathrm{Zr}$ de 0,8 ce qui est en accord avec la valeur proposée pour les sédiments marins non hydrothermaux (Marchig et al., 1982). Les dépôts et les sédiments hydrothermaux des dorsales océaniques sont très nettement enrichis en $\mathrm{Fe}$, $\mathrm{Cu}, \mathrm{Zn}, \mathrm{Cd}, \mathrm{Mn}, \mathrm{V}$ et $\mathrm{Pb}$ par rapport aux sédiments marins normaux. En revanche, ils ont des teneurs plus faibles en $\mathrm{Ni}$, en $\mathrm{Cr}$, en $\mathrm{Y}$ et en $\mathrm{Zr}$ (Fouquet et al., 1991 ; Hannington et al., 1991, 1995 ; Haymond et Kastner, 1981; Hekinian et Fouquet, 1985; Marchig et al., 1982 ; Metz et al., 1988 ; Turner et al., 1993). Les sédiments des fosses Atlantis II et Thétis se caractérisent par des teneurs élevées en certains métaux ( $\mathrm{Fe}, \mathrm{Mn}, \mathrm{Cu}$ et $\mathrm{Zn}$ ) et des concentrations en $\mathrm{Cr}$ et en $\mathrm{Zr}$ plus faibles que les sédiments biodétritiques (tableau I; figure 3; (Pierret, 1998) révélant leur nature hydrothermale.

Les sédiments de la fosse Atlantis II présentent les rapports $\mathrm{Cu} / \mathrm{Fe}$ (figure $2 a$ ), de l'ordre de ceux des sédiments hydrothermaux des rides océaniques plus matures, conjugués à des rapports $\mathrm{Ni} / \mathrm{Fe}$ les plus bas. L'activité hydrothermale importante dans la fosse Atlantis II est donc bien caractérisée par de forts rapports $\mathrm{Cu} / \mathrm{Fe}$ alliés à de faibles rapports $\mathrm{Ni} / \mathrm{Fe}$. L'échelle de cette première figure a été réduite dans le diagramme $2 \mathrm{a}^{\prime}$ afin de distinguer plus aisément le comportement des autres sédiments. Les échantillons des unités U1, U2 et U3 de la fosse Thétis, ainsi que ceux de la fosse Port-Soudan ont des rapports $\mathrm{Cu} / \mathrm{Fe}$ qui suggèrent une nature hydrothermale des sédiments, même si celle-ci est moindre que dans la fosse Atlantis II. Les sédiments de la fosse Chain sont dispersés car ils ont une large gamme de rapports $\mathrm{Ni} / \mathrm{Fe}\left(\right.$ figure $\left.2 a^{\prime}\right)$. Les rapports $\mathrm{Ni} / \mathrm{Fe}$ sont supérieurs 
à ceux des rapports $\mathrm{Cu} / \mathrm{Fe}$ sauf dans l'échantillon de $\mathrm{U} 3$ où les rapports $\mathrm{Cu} / \mathrm{Fe}$ et $\mathrm{Ni} / \mathrm{Fe}$ sont respectivement 11 et 2,3 . Or cette unité est la seule à avoir une nature hydrothermale, démontrée notamment par l'anomalie en $\mathrm{Eu}\left(\mathrm{Eu} / \mathrm{Eu}^{*}\right.$ normalisée par rapport au PAAS $=2,36$; Pierret, 1998 ; Pierret et al., 1998). En effet, les sédiments d'origine hydrothermale sont caractérisés par des anomalies positives en Eu due à la chimie des fluides hydrothermaux (Courtois et Treuil, 1977; German et al., 1997; Marchig et al., 1982 ; Michard et al., 1983; Mills et Elderfield, 1995). La figure $2 b$ représente l'évolution des rapports $\mathrm{Ni} / \mathrm{Fe}$ en fonction de l'anomalie en $\mathrm{Eu}$. Elle montre que les sédiments des fosses Atlantis II, Port-Soudan, Thétis et ceux de l'unité U2 de la fosse Chain sont caractérisés par les plus faibles rapports $\mathrm{Ni} / \mathrm{Fe}$ associés aux anomalies en $\mathrm{Eu}$ les plus importantes (tableau I), reflétant ainsi une influence hydrothermale. D'autre part les échantillons des fosses Atlantis II et Thétis, ainsi que ceux de l'unité U2 de la fosse Chain, se démarquent clairement des autres (zone grisée sur la figure 3) et révèlent leur nature hydrothermale par de faibles teneurs en $\mathrm{Zr}$ et la non corrélation entre $\mathrm{Cr}$ et $\mathrm{Zr}$. En effet, la forte sédimentation chimique liée dans ces fosses à l'arrivée de fluides minéralisés, dilue les apports détritiques. Par exemple, le taux de sédimentation est évalué à $200 \mathrm{~cm} 1000^{-1}$ ans dans le bassin occidental de la fosse Atlantis II (Bäcker et Schoell, 1972), alors qu'il n'est que de 3 à $4 \mathrm{~cm} 1000^{-1}$ ans ailleurs en mer Rouge (Rohling et al., 1998). La part de la sédimentation chimique sur la sédimentation biodétritique est très importante dans ce cas et les teneurs en $\mathrm{Zr}$ marqueur du détritique deviennent alors faibles dans les sédiments et ne sont plus corrélées au $\mathrm{Cr}$ apporté par les fluides hydrothermaux.

La nature hydrothermale des sédiments de la fosse Port-Soudan, en particulier de ceux des unités U2 et U3, a été clairement mis en évidence par les anomalies en $\mathrm{Eu}$ (figure $2 b$ ) et les rapports $\mathrm{Cu} / \mathrm{Fe}$ et $\mathrm{Ni} / \mathrm{Fe}$. Pourtant, la corrélation $\mathrm{Cr} / \mathrm{Zr}$ est excellente $(0,99)$ dans les sédiments de cette fosse et les teneurs sont proches de celles des sédiments biodétritiques. Cette corrélation implique que le $\mathrm{Cr}$ et le $\mathrm{Zr}$ ont la même source; ceci est envisageable si l'on suppose que le fluide hydrothermal qui s'épanche dans la fosse PortSoudan contient peu de $\mathrm{Cr}$ et de $\mathrm{Zr}$, et que ces éléments sont essentiellement apportés par du matériel détritique. Il a, en effet, été montré que la fosse Port-Soudan est alimentée par un fluide hydrother- mal qui a réagi avec le basalte à des températures n'excédant pas $150^{\circ} \mathrm{C}$ (Pierret, 1998), donc à de faibles profondeurs. Il semble alors que l'eau de mer n'a probablement pas atteint la zone de réaction où les métaux de la croûte sont lessivés (Alt, 1995). Le fluide résultant est alors vraisemblablement moins riche en métaux (et donc en $\mathrm{Cr}$ ) que les fluides qui se sont déchargés dans la fosse Atlantis II. La bonne corrélation entre $\mathrm{Zr}$ et $\mathrm{Cr}$ dans les sédiments de la fosse Port-Soudan est due aux faibles teneurs en $\mathrm{Cr}$ $\mathrm{du}$ fluide hydrothermal qui a alimenté la dépression et qui provient du matériel détritique. En effet, les sédiments des unités U2 et U3 de la fosse Port-Soudan contiennent 40 à $50 \%$ de silicates (argiles, quartz, plagioclases, tableau I; Pierret, 1998) et moins de carbonates que les sédiments normaux, probablement en partie dissous par la saumure acide. La fraction détritique étant plus importante, la corrélation est meilleure que pour les sédiments hors fosse.

Les points correspondants aux sédiments de la fosse Valdivia ont une position intermédiaire (figure $2 a^{\prime}$ ) et sont situés entre ceux de la carotte hors fosse (1016) et les sédiments hydrothermaux, avec des rapports $\mathrm{Cu} / \mathrm{Fe}(11$ à 23) supérieurs aux rapports $\mathrm{Ni} / \mathrm{Fe}(7,2$ à 18,3). Ainsi, bien que la minéralogie des sédiments de Valdivia soit proche de celle des sédiments biodétritiques de la mer Rouge, l'étude des rapports $\mathrm{Ni} / \mathrm{Fe}$ et $\mathrm{Cu} / \mathrm{Fe}$ laisse supposer une légère influence hydrothermale. Les analyses des boues de cette carotte sont peu dispersées sur le diagramme $\mathrm{Zr}-\mathrm{Cr}$ (figure 3), sans corrélation nette entre $\mathrm{Cr}$ et $\mathrm{Zr}\left(R^{2}=0,25\right)$ et avec des teneurs en $\mathrm{Zr}$ systématiquement inférieures à celles trouvées dans le sédiment de la carotte hors fosse pour des valeurs semblables en $\mathrm{Cr}$. Ce comportement pourrait être un indice en faveur d'une influence hydrothermale enregistrée dans les sédiments de la fosse Valdivia, mais celle-ci serait bien plus faible que pour les fosses Atlantis II et Thétis.

Les sédiments de la fosse Suakin ont un comportement singulier; ils se caractérisent par les rapports $\mathrm{Cu} / \mathrm{Fe}$ les plus faibles $(0,8$ à 6,9$)$ et les rapports $\mathrm{Ni} / \mathrm{Fe}$ les plus élevés (8 à 130 ; figure $2 a^{\prime}$ ) et ont un comportement opposé à ceux de la fosse Atlantis II. Ces observations confirment l'improbabilité d'une origine hydrothermale pour ces sédiments (Pierret et al., 2000), ce qui est conforté par les variations du rapport $\mathrm{Ni} / \mathrm{Fe}$ en fonction de l'anomalie en Eu (figure $2 b$ ). La corrélation $\mathrm{Cr} / \mathrm{Zr}$ est de 0,8 pour les sédi- 
ments de la fosse Suakin confirmant l'hypothèses d'une nature diagénétique sans apport hydrothermal (Pierret et al., 2000). Cependant, il reste à expliquer pourquoi les sédiments de la fosse Suakin, et dans une moindre mesure ceux de la fosse Chain, peuvent avoir des rapports $\mathrm{Ni} / \mathrm{Fe}$ si élevés, atteignant respectivement 130 et 35,2. Les sédiments marins classiques ont, en moyenne, des rapports $\mathrm{Ni} / \mathrm{Fe}$ plus faibles de l'ordre de 13 (Metz et al., 1988) et ceux de la carotte hors fosse (1016) représentant le fond biodétritique en mer Rouge, oscillent entre 6,2 et 24. Deux possibilités peuvent être envisagées pour expliquer les valeurs dans les sédiments de Suakin et Chain : (1) un processus a enrichi la phase solide en $\mathrm{Ni}$ (précipitation minérale, diagenèse), ou (2) une source externe a fourni plus de $\mathrm{Ni}$ que de $\mathrm{Cu}$. Cette seconde hypothèse semble la plus vraisemblable, car la source externe peut être constituée par les débris volcaniques observés dans ces fosses (Pierret, 1998; Pierret et al., 2000). Des basaltes et des produits volcaniques ont été analysés en mer Rouge (Altherr et al., 1988 ; Coleman et McGuire, 1988), et les rapports $\mathrm{Ni} / \mathrm{Fe}$ de ces composés sont élevés et varient de 4 à 80 , alors que les rapports $\mathrm{Cu} / \mathrm{Fe}$ compris entre 6 et 15 , sont de l'ordre de ceux des sédiments marins normaux. Ainsi, les verres qui se déposent dans les fosses, peuvent avoir été altérés, ce qui a pu libérer du Ni supplémentaire et donc entraîner une augmentation des rapports $\mathrm{Ni} / \mathrm{Fe}$. Du matériel éruptif a également été identifié dans les sédiments normaux de la mer Rouge (Boger et Faure, 1976; Boger et al., 1980), dont la carotte hors fosse 1016 (Pierret, 1998). Ceci pourrait expliquer pourquoi les rapports $\mathrm{Ni} / \mathrm{Fe}$ sont parfois plus élevés dans les sédiments biodétritiques de la mer Rouge que dans les sédiments marins habituels. Peu d'éléments permettent de discuter ici de la première hypothèse, qui reste une possibilité.

De même certains échantillons ont des teneurs importantes en $\mathrm{Cr}$ et en $\mathrm{Zr}$ (unité U3 de la fosse Chain et quelques sédiments des carottes 1013 et 1016, figure 3 ), toujours associées à de fortes teneurs en silicates et se trouvent dans les carottes qui contiennent des fragments de matériel éruptif. Or les basaltes et les verres peuvent être particulièrement riches en $\mathrm{Cr}$ (jusqu'à $1000 \times 10^{-6}$ ) et en $\mathrm{Zr}$ (jusqu'à $400 \times 10^{-6}$ ) (Altherr et al., 1988 ; Coleman et McGuire, 1988). La présence de tel matériel peut donc enrichir les sédiments en $\mathrm{Cr}$ et $\mathrm{Zr}$. Par exemple, l'unité U0 de la carotte 1032 de la fosse Atlantis II est formée de basalte altéré, et elle est la plus riche en $\mathrm{Si}$ (13 à $14 \%), \operatorname{Cr}\left(80\right.$ à $\left.100 \times 10^{-6}\right), \operatorname{Zr}\left(80\right.$ à $\left.110 \times 10^{-6}\right)$ et en silicates (argiles, plagioclases, feldspath potassique et pyroxène) de toute le carotte (tableau I). Ainsi, le lien entre $\mathrm{Cr}, \mathrm{Zr}$ et $\mathrm{Si}$ peut provenir d'une abondance de matériel détritique comme de matériel volcanique. La présence de débris éruptifs dans les carottes 1013 (fosse Suakin), 1016 (site hors fosse) et 1025 (fosse Chain) semble entraîner des enrichissements en certains éléments traces tels que $\mathrm{Ni}, \mathrm{Cr}$ et $\mathrm{Zr}$.

\section{CONCLUSION}

La nature hydrothermale des sédiments des fosses Atlantis II et Thétis est confirmée par de faibles rapports $\mathrm{Ni} / \mathrm{Fe}$ conjuguées à des rapports $\mathrm{Cu} / \mathrm{Fe}$ élevés, par de faibles teneurs en $\mathrm{Zr}$ et par l'absence de corrélation entre $\mathrm{Cr}$ et $\mathrm{Zr}$. Les sédiments de la fosse Port-Soudan, caractérisés par des rapports $\mathrm{Ni} / \mathrm{Fe}$ inférieurs et des anomalies positives en Eu supérieures à ceux des sédiments biodétritiques du site hors fosse, révèlent eux-aussi une nature hydrothermale. Cependant, celle-ci ne se traduit pas par une absence de corrélation entre $\mathrm{Cr}$ et $\mathrm{Zr}$, ceci peut s'expliquer par la nature du fluide hydrothermal ayant alimenté la fosse Port-Soudan et qui n'aurait pas circulé aussi profondément que dans les deux cas précédents (Thétis et Atlantis II). La fosse Suakin se caractérise par les plus faibles rapports $\mathrm{Cu} / \mathrm{Fe}$ et les plus forts rapports $\mathrm{Ni} / \mathrm{Fe}$, ainsi que par une corrélation positive entre $\mathrm{Cr}$ et $\mathrm{Zr}$. Ces résultats reflètent une absence d'activité hydrothermale dans cette fosse. Les sédiments de l'unité U3 de la carotte Chain présentent un faible rapport $\mathrm{Ni} / \mathrm{Fe}$ confirmant la nature hydrothermale de cette unité. Les rapports $\mathrm{Cu} / \mathrm{Fe}$ et $\mathrm{Ni} / \mathrm{Fe}$ et les teneurs en $\mathrm{Zr}$ et $\mathrm{Cr}$ des sédiments dans la fosse Valdivia suggèrent une faible participation de matériel d'origine hydrothermal.

Cette étude a donc mis en évidence une origine hydrothermale pour les sédiments des fosses Atlantis II, Thétis et de l'unité U3 de Chain. Les sédiments de la fosse Port-Soudan ont également enregistré une influence hydrothermale, mais celle-ci est moins importante que dans ceux des fosses Atlantis II et Thétis. Des débris éruptifs ont été observés dans les sédiments des carottes 1013, 1016 et 1025 et leurs présences pourraient expliquer les forts rapports $\mathrm{Ni}$ / $\mathrm{Fe}$ et les fortes concentrations en $\mathrm{Zr}$ et $\mathrm{Cr}$ enregistrés dans ces trois cas. 
Ainsi, les six fosses étudiées contiennent des sédiments de nature différente qui ont enregistré une signature hydrothermale variable d'un site à l'autre, sans véritable tendance géographique. En effet, l'activité la plus intense semble se situer au niveau de la fosse Atlantis II, alors que la fosse méridionale Suakin qui est la plus proche de la croûte océanique continue, contient des sédiments sans influence hydrothermale.

\section{Remerciements}

Nous remercions le capitaine et l'équipage du NO Marion Dufresne ainsi que Robert Rouault et Jean Samuel du Centre de géochimie de la surface (CNRS-ULP) pour leur aide technique.

\section{RÉFÉRENCES}

Alt, J.C., 1995. Subseafloor processes in Mid-Ocean Ridge hydrothermal systems. In: Seafloor hydrothermal systems: physical, chemical, biological and geological interactions, Geophysical Monograph by the American Geophysical Union, pp. 85-114.

Altherr, R., Henjes-Kunst, F., Puchelt, H., Baumann, A., 1988. Volcanic activity in the Red Sea axial trough-evidence for a large mantle diapir? Tectonophysics 150, 121-133.

Anschutz, P., Blanc, G., 1993a. L'histoire sédimentologique de la fosse Atlantis II (mer Rouge). Les apports de la micropaléontologie. C.R. Acad. Sci. Paris série II 317, 1303-1308.

Anschutz, P., Blanc, G., 1993b. Le rapport NaCl/eau des boues minéralisée de la fosse Atlantis II (mer Rouge). calcul de la teneur en halite des sédiments et implication sur la paléotempérature du milieu. C.R. Acad. Sci. Paris série II 317, 15951600.

Anschutz, P., Blanc, G., 1995a. Chemical mass balances in metalliferous deposits from the Atlantis II Deep, Red Sea. Geochim. Cosmochim. Acta 59, 4205-4218.

Anschutz, P., Blanc, G., 1995b. Diagenetic evolution of the DOP facies from the Atlantis II Deep (Red Sea): Evidence of early hydrothermal activity. Oceanol. Acta 18, 105-112.

Bäcker, H., Schoell, M., 1972. New deeps with brines and metalliferous sediments in the Red Sea. Nature Phys. Sci. 240, 153158.

Bäcker, H., Richter, H., 1973. Die rezente hydrothermal-sedimentäre Lagestätte Atlantis II Tief im Roten Meer. Geol. Rundsch. 62, 697-741.

Blanc, G., Anschutz, P., Pierret, M.C., 1998. Metalliferous sedimentation in the Atlantis II Deep: a geochemical insight. In: Purcer B.H., Bosence D.W.J. (Eds.), Sedimentation and Tectonics of Rift Basins: Red Sea-Gulf of Aden. Chapman \& Hall, London, pp. 510-524.
Blanc, G., 1994. Geochemical studies on selected sediment samples from the Lau Backarc Basin: evidence for hydrothermal ponded sediments. Proc. Ocean Drilling Program 135, 689707.

Boger, P.D., Faure, G., 1976. Systematic variations of sialic and volcanic detritus in piston cores from the Red Sea. Geochim. Cosmochim. Acta 40, 731-742.

Boger, P.D., Boger, J.L., Faure, G., 1980. Systematic variation of ${ }^{87} \mathrm{Sr} /{ }^{86} \mathrm{Sr}$ ratios, $\mathrm{Sr}$ compositions, selected major-oxide concentrations, and mineral abondance in piston cors from the Red Sea. Chem. Geol. 29, 13-38.

Bruland, K.W., Franks, R.P., 1983. Mn, Ni, Cu, Zn and Cd in the Western North Atlantic. In: Wong, C.S., Boyle, E., Bruland, K.W., Burton, J.D., Goldberg, E.D. (Eds.), Trace Metale in Sea Water. Plenum Press, New York.

Calvert, S.E., Pedersen, T.F., 1993. Geochemsitry of recent oxic and anoxic marine sediments: Implications for the geological record. Mar. Geol. 113, 67-88.

Chiba, H., 1995. Chemical modeling of seawater-rock intercation: Effect of rock-type on the fluid chemistry and mineral assemblage. In: Sakai, H., Nozaki, Y. (Eds.), Biogeochemical Processes and Ocean Flux in the Western Pacific. Terra Scientific Publishing Company, Tokyo, pp. 469-486.

Courtois, C., Treuil, M., 1977. Distribution des terres rares et de quelques éléments traces dans les sédiments récents des fosses de la mer Rouge. Chem. Geol. 20, 57-72.

Coleman, R.G., McGuire, A.V., 1988. Magma systems related to the Red Sea opening. Technonophysics 150, 77-100.

Collier, R.W., Edmond, J.M., 1983. Plankton composition and trace metal fluxes from the surface ocean. In: Trace Metals in Seawater, NATO Conf. Ser 4, Vol. 9, Plenum, New York, pp. 789-890.

Degens, E.T., Ross, D.A., 1969. Hot brines and recent heavy metal deposits in the Red Sea. Springer Verlag, New York.

Fouquet, Y., Von Stackelberg, U., Charlou, J.L., Donval, J.P., Erzinger, J., Foucher, J.P., Herzig, P., Mühe, R., Soakai, S., Wiedicke, M., Whitechurch, H., 1991. Hydrothermal activity and metallogenesis in the Lau back-arc basin. Nature 349, $778-781$.

German, C.R., Bourlés, D.L., Brown, E.T., Hargt, J., Colley, S., Higgs, N.C., Ludford, E.M., Nelsen, T.A., Feely, R.A., Raibeck, G., Yiou, F., 1997. Hydrothermal scavenging on the Juan de Fuca Ridge: ${ }^{230}$ Thxs, ${ }^{10} \mathrm{Be}$ and REEs in ridge-flanks sediments. Geochim. Cosmochim. Acta 61, 4067-4078.

Hannington, M.D., Herzig, P.M.., Scott, S.D., Thompson, G., Rona, R.A., 1991. Comparative mineralogy and geochemistry of gold-bearing sulfide deposits on the mid-ocean ridges. Mar. Geol. 101, 217-248.

Hannington, M.D., Jonasson, I.R., Herzig, P.M., Petersen, S., 1995. Physical and chemical processes of seafloor mineralization at Mid-Ocean Ridges. In: Seafloor Hydrothermal Systems; Physical, Chemical, Biological and Geological Interactions. Geophysical Monograph 91. American Geophysical Union, pp. $115-157$. 
Haymond, R.M., Kastner, M., 1981. Hot spring deposits on the East Pacific Rise at $21^{\circ} \mathrm{N}$ : preliminary description of mineralogy and genesis. Earth Planet. Sci. Lett. 53, 363-381.

Hekinian, R., Hoffert, M., Larqué, P., Cheminée, J.L., Stoffers, P., Bideau, D., 1993. Hydrothermal $\mathrm{Fe}$ and $\mathrm{Si}$ oxyhydroxide deposits from south Pacific intraplate volcanoes and East Pacific Rise axial and off-axial regions. Econ. Geol. 88, 2099-2121.

Hekinian, R., Fouquet, Y., 1985. Volcanism and metallogenesis of axis and off-axial structures on the East Pacific Rise near $13^{\circ}$ N. Econ. Geol. 80, 221-249.

Marchig, V., Gundlach, H., Möller, P., Schley, F., 1982. Some geochemical indicators for discrimination between diagenetic and hydrothermal metalliferous sediments. Mar. Geol. 50, $241-256$.

Metz, S., Trefry, J.H., Nelsen, T.E., 1988. History and geochemistry of a metalliferous sediment core from the Mid-Atlantic Ridge at $26^{\circ} \mathrm{N}$. Geochim. Cosmochim. Acta 52, 2369-2378.

Michard, A., Albarède, F., Michard, G., Minster, J.F., Charlou, J.L., 1983. Rare-earth elements and uranium in high-temperature solutions from East Pacific Rise hydrothermal vent field $\left(13^{\circ} \mathrm{N}\right)$. Nature 303, 795-797.

Mills, R., Elderfield, H., 1995. Rare earth element geochemistry of hydrothermal deposits from the active TAG Mound, $26^{\circ} \mathrm{N}$ Mid-Atlantis Ridge. Geochim. Cosmochim. Acta 59, 35113524.

Pierret, M.C., 1998. Les saumures et les sédiments de sept fosses de l'axe de la mer Rouge $\left(19-23^{\circ} \mathrm{N}\right)$. Etude minéralogique, géochimique et isotopique. Rôle de l'hydrothermalisme. Thèse, Université Louis Pasteur, Strasbourg, 352 p. (en dépôt à la soc. géol. France).
Pierret, M.C., Blanc, G., Bosch, D., 1998. Sr, Pb isotopes and REE analyses of five cores of Red Sea. An insight into hydrothermal input, 8th Goldsmith Conference. Miner. Mag. 62, 1176-1177.

Pierret, M.C., Blanc, G., Clauer, N., 2000. Sur l'origine de la pyrite framboïdale dans les sédiments de la fosse Suakin (mer Rouge). C.R. Acad Sci. 330, 1-8.

Pottorf, R.J., Barnes, H.L., 1983. Mineralogy, geochemistry, and ore genesis of hydrothermal sediments from the Atlantis II Deep, Red Sea. Econ. Geol. Monogr. 5, 198-223.

Rohling, E.J., Fenton, M., Jorissen, F.J., Bertrand, P., Ganssen, G., Caulet, J.P., 1998. Magnitudes of sea-level lowstands of the past 500,000 years. Nature 394, 162-165.

Samuel, J., Rouault, R., Bensnus, Y., 1985. Analyse multiélémentaire standardisée des matériaux géologiques en spectrométrie d'émission par plasma à couplage inductif. Analusis 13, 312317.

Turner, R.J.X., Ames, D.E., Franklin, J.M., Goodfellow, W.D., Leitch, C.H.B., Höy, T., 1993. Character of active hydrothermal mounds and nearby altered hemipelagic sediments in the hydrothermal areas of middle valley, northern Juan de Fuca Ridge: data on shallow cores. Can. Mineralog. 31, 973-995.

Von Damm, K.L., 1995. Controls on the chemistry and temporal variability of seafloor hydrothermal fluids. In: Humphris, S.E., Zierenberg, R.A., Mullineaux, L.S., Thomson, R.E (Eds.), Seafloor Hydrothermal Systems: Physical, Chemical, Biological, and Geological interactions. American Geophysical Union. Geophysical Monograph 91, pp. 222-247.

Zierenberg, R.A., Schanks, W.C. III, 1986. Isotopic contraints on the origin of the Atlantis II, Suakin and Valdivia brines, Red Sea. Geochim. Cosmochim. Acta 50, 2205-2214. 